\title{
Intensity modulated dose calculation with an improved experimental pencil-beam kernel
}

\author{
Juan Diego Azcona ${ }^{a)}$ \\ Department of Oncology, Division of Radiation Physics, Clínica Universidad de Navarra, \\ Avda. Pío XII, 36 Pamplona, Navarra 31008, Spain \\ Javier Burguete \\ Department of Physics and Applied Mathematics, Facultad de Ciencias, Universidad de Navarra, \\ C/Irunlarrea s/n, Pamplona, Navarra 31008, Spain
}

(Received 17 November 2009; revised 16 July 2010; accepted for publication 16 July 2010; published 11 August 2010)

Purpose: This article presents an improved pencil-beam dose calculation formalism based on an experimental kernel obtained by deconvolution. The new algorithm makes it possible to calculate the absorbed dose for all field sizes.

Methods: The authors have enhanced their previous work [J. D. Azcona and J. Burguete, Med. Phys. 35, 248-259 (2008)] by correcting the kernel tail representing the contribution to the absorbed dose far from the photon interaction point. The correction was performed by comparing the calculated and measured output factors. Dose distributions and absolute dose values calculated using the new formalism have been compared to measurements. The agreement between calculated and measured dose distributions was evaluated according to the $\gamma$-index criteria. In addition, 35 individual intensity-modulated radiation therapy (IMRT) fields were calculated and measured in polystyrene using an ionization chamber. Furthermore, a series of 541 IMRT fields was calculated using the algorithm proposed here and using a commercial IMRT optimization and calculation software package. Comparisons were made between the calculations at single points located at the isocenter for all the beams, as well as between beams grouped by anatomic location.

Results: The percentage of points passing the $\gamma$-index criteria $(3 \%, 3 \mathrm{~mm})$ when comparing calculated and measured dose distributions is generally greater than $99 \%$ for the cases studied. The agreement between the calculations and the experimental measurements generally lies in the $\pm 2 \%$ interval for single points, with a mean value of $0.2 \%$. The agreement between calculations using the proposed algorithm and using a commercial treatment planning system is also between $\pm 5 \%$.

Conclusions: An improved algorithm based on an experimental pencil-beam kernel obtained by deconvolution has been developed. It has been validated clinically and promises to be a valuable tool for IMRT quality assurance as an independent calculation system for monitor units and dose distributions. An important point is that the algorithm presented here uses an experimental kernel, which is therefore independent of Monte-Carlo-calculated kernels. (c) 2010 American Association of Physicists in Medicine. [DOI: 10.1118/1.3476467]

Key words: deconvolution, Hankel transform, intensity modulation, IMRT treatment plan verification, pencil beam kernel

\section{INTRODUCTION}

The pencil-beam approach is frequently used in dose calculations for external-beam radiotherapy and intensitymodulated radiation therapy (IMRT). In homogeneous media, it maintains the accuracy of the three-dimensional convolution-superposition algorithm while increasing speed, because the pencil-beam kernels can be viewed as the convolution over a straight line of a series of point dose kernels properly weighted along the depth according to their fluence attenuation. In IMRT planning, the pencil-beam approach is normally used during the optimization process, as well as for the final dose calculations in a region without severe inhomogeneities, such as for head radiosurgery techniques. An additional application of the pencil-beam algorithm is as an independent system for checking treatment planning system (TPS) calculations.

Independent verification of monitor units and dose distributions is a common task in IMRT quality assurance (QA). Verification of calculated doses is an important issue and experimental measurements are the most reliable way to check them. However, measurements are time-consuming and once an IMRT program is well established, replacement of measurements by independent calculations can be considered. This substitution makes it possible to preserve linearaccelerator time for treating patients that otherwise would be dedicated to QA.

A number of different models for independent calcula- 
tions have been developed in recent years that calculate doses at single points or as two-dimensional dose distributions. ${ }^{1-5}$ They use tissue-phantom ratio values ${ }^{1-3}$ or a pencil-beam approach, where the kernels have been empirically fitted to mathematical functions ${ }^{4}$ or modeled using Monte Carlo methods. ${ }^{5}$

In a previous study, ${ }^{6}$ the authors presented a pencil-beam approach that enables the calculation of two-dimensional dose distributions. This approach is based on an experimental kernel obtained by deconvolution. Therefore, its calculations are completely independent of those performed by commercial TPS, which are usually based on Monte-Carlogenerated kernels. However, this method is limited to small fields. The experimental technique relies on a circular lead collimator that cannot be made larger without modifying the linac head accessory mount. It is therefore impossible to obtain information for radii larger than $7 \mathrm{~cm}$. On the other hand, because the central-kernel drop was properly characterized, the relative dose maps were found to agree well with measurements for small segments of highly modulated fields.

In this study, this algorithm is improved by correcting the kernel tail to calculate the absorbed dose properly for all field sizes. With this improvement, the output-factor curve can be fully reconstructed. Seven two-dimensional measured dose distributions are compared to the calculation results using the $\gamma$-index method. ${ }^{7}$ Comparisons of calculations and measurements are also presented in terms of absolute dose values at single points for 35 beams. Finally, in the context of a clinical application, point dose calculations for 541 beams are compared to calculations using a commercial TPS. Because the deconvolved kernel was obtained by a procedure independent of Monte Carlo calculations, it is a suitable independent tool for verifying TPS dose calculations. These data demonstrate that this improved algorithm can be used for dose calculation and independent verification in IMRT treatment planning.

\section{MATERIALS AND METHODS}

\section{II.A. Deconvolution of the kernel using the Hankel transform}

The deconvolution procedure for the extraction of pencilbeam kernels from dose measurements was initially explored by Chui and Mohan. ${ }^{8}$ They assumed that the kernel could be expressed as the product of two components, each representing the behavior of the kernel in one of two orthogonal directions. They used a fast Fourier transform to obtain these functions from dose measurements and assumed that the fluence could be modeled as a two-dimensional step function. This approach has certain limitations that have been discussed elsewhere and that motivated the authors to develop a new method for deconvolving the kernel. ${ }^{6}$

In the model presented in Ref. 6 and improved here, the pencil-beam kernel was obtained using a deconvolution procedure with dose measurements in a circular field $50 \mathrm{~mm}$ in diameter and modeling the incident fluence as a $2 \mathrm{D}$ circular step function. The Hankel transform was used to obtain the kernel. The Hankel transform can be derived from the Fou- rier transform for the 2D case when there is rotational symmetry. This enables characterization of the two-dimensional behavior of the kernel. As in the Fourier case, the Hankel transform converts convolutions into products. Denoting the Hankel transform by $H$, the absorbed dose by $D$, the energy fluence by $\Psi$, and the kernel by $k$,

$$
H(D)=H(\Psi \otimes k)=H(\Psi) \times H(k),
$$

so

$$
k=H\left[\frac{H(D)}{H(\Psi)}\right],
$$

because the Hankel transform is self-inverse $\left(H^{-1}=H\right)$.

An experimental approach used in this way should take into account two important aspects of small-field dose calculations and therefore of IMRT dose calculations: The lateral range of the secondary electrons and the partial obscuring of the primary source. These two effects are responsible for the physical and geometrical penumbra seen on the dose profiles and affect the field output. ${ }^{9,10}$ Recent publications have studied pencil-beam characterization in the context of the small fields frequently used in IMRT ${ }^{11,12}$ and in the context of IMRT optimization. ${ }^{13,14}$

\section{II.B. Dose calculation using the enhanced algorithm}

The algorithm used here has been extended on the basis of previous work ${ }^{6}$ and differs from it in several ways. The absorbed dose is calculated using the following expression:

$$
\begin{aligned}
D\left(x, y, z_{0}\right)= & D_{\mathrm{MU}}^{\mathrm{ref}} \mathrm{MU}[\Psi \otimes k]=D_{\mathrm{MU}}^{\mathrm{ref}} \mathrm{MU} \\
& \times \iint_{S} \Psi\left(x^{\prime}, y^{\prime}\right) k\left(x-x^{\prime}, y-y^{\prime}, z_{0}\right) d x^{\prime} d y^{\prime},
\end{aligned}
$$

where $\Psi$ denotes the energy fluence, $k$ is the pencil-beam kernel at a specific depth $z_{0}, D_{\mathrm{MU}}^{\text {ref }}$ is the calibration factor of the machine at the reference conditions, and MU is the number of monitor units. Because the energy fluence can be viewed as the convolution of a primary fluence coming from a point source $\Psi^{\prime}$ with that from an extended source $s_{e}$, it is possible to rewrite Eq. (3) as

$$
\begin{aligned}
D\left(x, y, z_{0}\right) & =D_{\mathrm{MU}}^{\mathrm{ref}} \mathrm{MU}[\Psi \otimes k]=D_{\mathrm{MU}}^{\mathrm{ref}} \mathrm{MU}\left[\Psi^{\prime} \otimes s_{e} \otimes k\right] \\
& =D_{\mathrm{MU}}^{\mathrm{ref}} \mathrm{MU}\left[\Psi^{\prime} \otimes w\right],
\end{aligned}
$$

where $w=s_{e} \otimes k$ is the kernel obtained by deconvolution. ${ }^{6}$ The term $s_{e}$ includes the characterization of the finite extent of the primary source as well as that of the head scatter source. Although the algorithm provides a function $w$ that is not exactly the kernel $k$, this approach enables dose calculations for any fluence distribution. The kernel $w$ reproduces the geometrical and physical penumbra and its convolution with the energy fluence $\Psi^{\prime}$ reproduces the output-factor curve.

The energy fluence coming from a point source $\Psi^{\prime}$ is modeled on the basis of the file sent from the TPS to the linear accelerator to perform treatment using the step-and- 
shoot technique. From the leaf positions, the modulation index is calculated according to the definition by Xing et $a l^{2}$ The index includes the effects of the jaws and of multileaf collimator intraleaf transmission. Minor corrections due to tongue-and-groove effects and interleaf transmission are performed according to Chui et al. ${ }^{5}$

Modeling the fluence as the value of the modulation index assumes a flat open-beam energy-fluence distribution. The energy fluence $\Psi^{\prime}$ should take into account any deviations from a flat distribution. Actually, the open-beam energyfluence distribution has a shape that deviates from a step function and exhibits "horns." To represent lateral energyfluence variations, the authors measured the open-beam energy-fluence distribution $\Psi_{\text {op }}^{\prime}$ using a diode on a shallow depth of water $(5 \mathrm{~mm})$ for the maximum field size available: A $40 \times 40 \mathrm{~cm}^{2}$ field. The modulation index is then multiplied by the open-beam energy-fluence distribution $\Psi_{\mathrm{op}}^{\prime}$ to obtain $\Psi^{\prime}$. This open-beam energy-fluence distribution represents the lateral variations in the energy fluence which are assumed to come from a point source and its value is relative to a value of 1.000 at the central axis. This approximation can be performed provided that for a such a large field (ideally infinite), the flattening filter can be completely seen from all points in the lateral profile, so that the relative fluence distribution can be assumed to be unaffected in the in-field area. Changes in the geometric penumbra with field size due to head scatter are taken into account by means of the extended source term $s_{e}$.

The convolution integral in Eq. (4) enables calculation of the relative dose distribution. The kernel $w$ reproduces the output-factor curve, which is normalized for a field size of $10 \times 10 \mathrm{~cm}^{2}$. Using the term $D_{\mathrm{MU}}^{\mathrm{ref}}$, which is the reference dose rate for a $10 \times 10 \mathrm{~cm}^{2}$ field at the depth of calculation, the distribution can be related to absolute dose values.

\section{II.C. Correction of the kernel and reconstruction of the output-factor curve}

In the pencil-beam approach, the output-factor curve can be reconstructed by convolving the kernel obtained with a fluence modeled from the dimensions of each field. This was achieved in previous work, ${ }^{6}$ but a "saturation" effect was seen for field sizes of $7 \mathrm{~cm}$ and above (see Fig. 1, dashed line).

The problem that made the output-factor curve fail occurred at the kernel tail. This problem arose because of two technical limitations of the previous approach to processing the dose-distribution signal. The most important limitation was the size of the lead collimator which, in the system used, cannot be much larger than $10 \mathrm{~cm}$. The second one is that the film used had an area of $15 \times 15 \mathrm{~cm}^{2}$. These two effects combined prevented the authors from taking into account the dose absorbed at larger radii and therefore from reproducing the kernel tail properly. When this kernel was used to reconstruct the output-factor curve, the contribution to the absorbed dose at the center of the field by the photon beam interacting at large distances was not correct, so that these output factors for large fields were underestimated. The

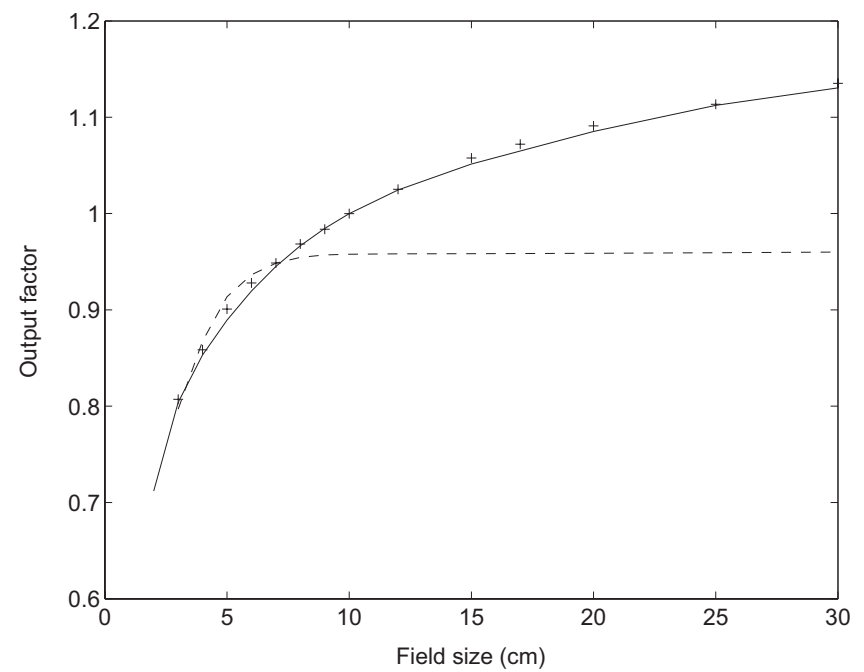

FIG. 1. Experimental output factors measured (crosses) compared to the calculated curve with the corrected kernel (solid line) and the uncorrected kernel (dashed line). The normalization is done for the $10 \times 10 \mathrm{~cm}^{2}$ field size. (To be compared to Ref. 6, Fig. 2.) The output-factor curve up to the $30 \times 30 \mathrm{~cm}^{2}$ field size is presented. The size of the segments used in IMRT is smaller.

central-kernel drop, on the other hand, was already properly characterized, so the relative distributions were in good agreement with the measurements, especially for highly modulated fields.

In this study, the drawback just described was overcome by correcting the kernel tail. The experimental output-factor curve was used to obtain the behavior of the kernel for all field sizes. The final kernel is determined by two kinds of experimental measurements. For $r<r_{0}$, the previous formalism was used. For $r>r_{0}$, the output factors were used to make the correction and overcome the limitations of the previous algorithm.

An important question is at what radius $r_{0}$ the correction should begin. The authors obtained kernels with the correction beginning at $r_{0}=17,28$, and $39 \mathrm{~mm}$. The value of the kernel at $r_{0}$ is $0.3 \%$ or less of the value at the center; for larger radii, the value of the kernel decreases. For radii smaller than the starting point, the output factors are properly reproduced. This can be fully explained by considering that the output-factor curve was properly reproduced for field sizes smaller than $7 \times 7 \mathrm{~cm}^{2}$ (Fig. 1), where the signal was properly processed. Because the output-factor curve begins to saturate at field sizes of $7 \times 7 \mathrm{~cm}^{2}$, it is unnecessary to check the effect of corrections beginning at radii larger than $39 \mathrm{~mm}$.

For radii larger than $r_{0}$, the experimental and calculated output factors differ and the difference increases with field size (Fig. 1). The authors have corrected the tail of the kernel, which is responsible for this difference, using a recursive procedure. With a resolution of $\Delta r=2 \mathrm{~mm}$, the kernel is corrected so that it can reconstruct the experimental output factor up to $r_{i+1}=r_{i}+\Delta r$. Then the next output factor is calcu- 
lated using the improved kernel. The effect of leaf transmission is taken into account in the recursive procedure when calculating the convolution.

The experimental output-factor curve is fitted using a fifth-order polynomial equation. Because the kernel has rotational symmetry, the output factor reconstructed for circular fields is assumed to be that of the equivalent square field for purposes of looking up the experimental output-factor curve.

\section{II.D. Setup for evaluation of the improved algorithm}

\section{II.D.1. Comparison between calculated and measured dose distributions}

To test the improved algorithm, a series of dosedistribution measurements was collected. The seven intensity-modulated fields reported in Ref. 6 were recalculated and compared against measurements from radiographic films. The KonRad IMRT optimization and planning system (Siemens Medical Systems Oncology Care Systems, USA) was used to obtain the IMRT fields. KonRad uses a pencilbeam algorithm for optimization and final dose calculation. The optimization is based on the gradient search method. The fields were delivered using the step-and-shoot technique and measured on a Mevatron Primus linear accelerator (Siemens Medical Systems Oncology Care Systems, USA). The film used was Kodak XOmatV, located at $15 \mathrm{~cm}$ depth in the polystyrene phantom RW3 from PTW (Freiburg, Germany), model 29672. The phantom consists of a series of slabs of $30 \times 30 \mathrm{~cm}^{2}$ and $1 \mathrm{~cm}$ thickness. Several slabs (at least ten) are located below the detector to provide full backscattering conditions. The film was placed orthogonally to the beam axis and at the isocenter (SAD). The calculations were performed using the same setup.

The dose distributions were compared using the $\gamma$ index, ${ }^{7}$ with tolerance criteria of $3 \%$ for dose difference and $3 \mathrm{~mm}$ for distance to agreement.

\section{II.D.2. Comparison between calculated and measured absolute dose values at single points}

A series of single-point comparisons between calculations and measurements under the same geometrical conditions was also made. All IMRT fields were delivered using the step-and-shoot technique. The depth chosen was again $15 \mathrm{~cm}$ in polystyrene and at the isocenter (detector and calculation plane located at isocenter SAD). This depth is close to typical depth values in IMRT cases, where many fields are used to treat deep tumors. The ionization chamber used to measure the absolute values of the absorbed dose was a PTW 31002 (PTW Freiburg, Germany). It has a nearly spherical volume of $0.125 \mathrm{~cm}^{3}$ and is commonly used in IMRT verification. The same polystyrene phantom as before was used to obtain the measurements. The linear accelerators used at the authors' institution are a Mevatron Primus and an Oncor (Siemens Medical Systems Oncology Care Systems, USA). The energy spectra of both accelerators are very similar, as well as their geometrical designs and head dimensions, and therefore the same kernel, originally derived for the Primus, was used in all the calculations for both linacs. The energy used in these cases was $15 \mathrm{MV}$ photons, a value typically used in the authors' institution for IMRT treatments. The $\mathrm{TPR}_{20,10}$ is 0.763 for the Primus and 0.764 for the Oncor. The calculations using the proposed algorithm were performed in polystyrene.

Thirty-five IMRT fields were compared. At the measurement point, which is always located at the isocenter, a procedure analogous to the $\gamma$-index method was used to prevent situations with high-dose gradients. The experimental values were compared to a $4 \times 4$-point calculated dose matrix centered at the isocenter and the point of best agreement was selected. The largest distance between points was $2.12 \mathrm{~mm}$. The resolution of the calculation grid with the proposed algorithm was $1 \mathrm{~mm}$. Furthermore, it was ensured that the dose signal in the ionization chamber was strong enough to make an accurate measurement. This check avoided incorrect dose estimates because of differences in spatial extent between the calculation grid and the measurement volume.

The corresponding data calculated with the KonRad TPS and their differences from the measurements are also presented here. With the KonRad, the grid resolution used for optimization and dose calculations is $3 \mathrm{~mm}$. This setup represents the usual conditions under which the KonRad is used for optimization and dose calculations. The $3 \mathrm{~mm}$ grid spacing provides a good compromise between speed and accuracy. The purpose is not to test the KonRad against the independent calculations, but rather to compare the latter to the measurements.

\section{II.D.3. Comparison of doses at single points calculated with the proposed algorithm and with a commercial TPS}

Calculations with the TPS and the proposed algorithm were performed using the usual grid size for each method. This approach provided an evaluation of the use of the proposed algorithm as an independent calculation tool and highlighted the useful information it provides because it performs a fast two-dimensional dose calculation on a $1 \mathrm{~mm}$ resolution grid.

The results of the proposed algorithm and those obtained from the KonRad TPS in a homogeneous medium (polystyrene) were then compared. The IMRT fields are composed of a set of segments (step-and-shoot) and each field is incident orthogonally to the polystyrene phantom described above. The calculation plane is at the isocenter and at $15 \mathrm{~cm}$ depth. This paper presents the differences observed between the two sets of calculations in 541 fields, corresponding to 79 treatment plans used with patients. These plans correspond to various types of tumors at various treatment sites, including pelvic (rectum, prostate, bladder, gynecologic, and colon), thoracic (mediastinum and breast), and the head and neck region (brain and head and neck tumors). The differences are also presented separately for each treatment site and type of tumor. 
TABLE I. Percentage of points passing $\gamma$ criteria for seven cases considered in Ref. 6, calculated with the uncorrected and corrected kernel. The cases with the largest segments (4 and 6) have the most important improvement.

\begin{tabular}{ccc}
\hline \hline & \multicolumn{2}{c}{$\%$ of points passing $\gamma$ criteria } \\
\cline { 2 - 3 } Case & Uncorrected kernel & Corrected kernel \\
\hline 1 & 99.05 & 99.28 \\
2 & 99.89 & 99.76 \\
3 & 99.71 & 99.83 \\
4 & 84.75 & 97.94 \\
5 & 99.60 & 99.51 \\
6 & 97.60 & 99.84 \\
7 & 99.70 & 99.94 \\
\hline \hline
\end{tabular}

Another comparison is provided between the mean difference for all the step-and-shoot fields that correspond to the same treatment plan in a patient, as calculated using the proposed algorithm and as calculated by the KonRad system. The purpose of this comparison is to evaluate the overall difference in the calculation of monitor units for the isocenter at a phantom with several beams incident (assuming a constant depth of calculation for all beams and a flat surface of incidence).

\section{RESULTS}

\section{III.A. Derivation of a kernel valid for all fields}

The kernel was corrected beginning at radii $r_{0}$ of 17, 28, and $39 \mathrm{~mm}$. These corrections yielded a kernel that properly describes the experimental output-factor curve. Moreover, the dose profiles are very similar with the three corrections. The following discussion focuses on the $17 \mathrm{~mm}$ kernel and the remaining results presented are calculated using this kernel. Similar results can be obtained using the other kernels.

Figure 1 shows a comparison of the reconstructed and measured output-factor curves. The new curve based on the corrected kernel is also compared to that calculated based on the uncorrected kernel. Note that the output-factor curve is calculated for fields up to the maximum available in the accelerator $\left(40 \times 40 \mathrm{~cm}^{2}\right)$. The formalism is general. In IMRT, however, the fields used are smaller and the segments that constitute each field even more so.

\section{III.B. Evaluation of the improved algorithm}

\section{III.B.1. Dose distributions compared according to the $\gamma$ index}

The $\gamma$-index calculation for representing the agreement between calculated and measured values was used for the same modulated fields reported in Ref. 6 (see Table I). With the corrected kernel, the $\gamma$ is clearly improved.

The percentage of points with $\gamma>1$ is less than $0.7 \%$ in all cases except case $4(2.06 \%)$. With respect to $\gamma>1.5$, in all cases, the percentage of points not fulfilling the criteria is less than $0.09 \%$, except for case $4(0.15 \%)$. This case is the combination of the largest segments in the fields studied and was poorly calculated by the previous algorithm. These re- sults represent a clear improvement over the calculations performed with the uncorrected kernel, for which the $\gamma$ results were reported in Ref. 6 (see Table I). With the corrected kernel, the percentage of points that pass the $\gamma$ criteria is higher.

In addition to algorithm accuracy, calculation speed is also important. The time required to calculate seven $2 \mathrm{D}$ dose maps orthogonal to the beam axis at a fixed depth for a seven-field IMRT plan with a $1 \mathrm{~mm}$ grid resolution is less than 1 min on a $3.06 \mathrm{GHz}$ Pentium 4 processor. The proposed algorithm takes advantage of the calculation speed of the fast Fourier transform to perform the calculations for all the beams in a treatment plan. This capability can be used to provide a fast calculation in a high-resolution grid for IMRT dose-distribution and monitor-unit verification.

\section{III.B.2. Absolute dose values at single points}

The differences between calculated and measured values are presented in Table II. These differences were calculated according to the following formula:

$$
\% \text { dif }=\frac{D_{\text {cal }}-D_{\text {meas }}}{D_{\text {Max Map }}}
$$

where $D_{\text {cal }}$ represents the calculated dose (independent algorithm or KonRad), $D_{\text {meas }}$ is the measured dose, and $D_{\text {Max Map }}$ is the maximum value in the dose map calculated with KonRad. In this way, the difference is related to a high-dose point. In an open radiation beam, there are different areas (central axis, in-field, out of the field, buildup, and penumbra), and in each area, the achievable accuracy for the dose calculation is different. ${ }^{15,16}$ The recommended tolerances also vary between areas. In IMRT, the situation is even more complicated because at many calculation points, there are contributions from transmitted radiation, indirect radiation, or penumbra. In the interests of uniformity, the differences between calculated and measured values have been related to the maximum dose level in the dose distribution. Using this criterion, differences in low-dose areas, where a small absolute difference can represent a large percentage change, are related to a larger value to calculate a relative difference. These low-dose areas are usually of less clinical importance, so this manner of calculating the relative difference is justified.

The mean of the differences between measured values and TPS calculated values is $1.1 \%$, in an interval between $-3.4 \%$ and $7 \%$. With the proposed algorithm, the mean of these differences is $0.1 \%$, in an interval between $-2.9 \%$ and $3.8 \%$.

The agreement between calculated and measured values is good. The experimental validation and comparison with measured values is the most important part of this research. A plot of the differences between the values calculated by the proposed algorithm and the measured values is shown in Fig. 2. 
TABLE II. Measured values of the absolute dose values in the isocenter of several modulated fields and comparison with the calculated values with the TPS and our algorithm.

\begin{tabular}{|c|c|c|c|c|c|c|}
\hline \multirow[b]{2}{*}{ Field } & \multicolumn{4}{|c|}{$\begin{array}{l}\text { Dose } \\
\text { (cGy) }\end{array}$} & \multicolumn{2}{|c|}{$\begin{array}{c}\text { Diff. } \\
(\%)\end{array}$} \\
\hline & TPS & Ind. alg. & Meas. & Max & Meas.-TPS & Meas.-ind. alg. \\
\hline 1 & 26 & 23.8 & 23.9 & 54 & 3.9 & -0.2 \\
\hline 2 & 86 & 81.7 & 83 & 87 & 3.4 & -1.5 \\
\hline 3 & 83 & 76.2 & 77.7 & 83 & 6.4 & -1.8 \\
\hline 4 & 38 & 32.9 & 32.6 & 77 & 7.0 & 0.4 \\
\hline 5 & 16 & 16.5 & 15.7 & 57 & 0.5 & 1.4 \\
\hline 6 & 42 & 41.8 & 41.8 & 58 & 0.3 & 0.0 \\
\hline 7 & 53 & 50.4 & 48.3 & 108 & 4.4 & 1.9 \\
\hline 8 & 31 & 31.7 & 31.7 & 109 & -0.6 & 0.0 \\
\hline 9 & 54 & 53.6 & 53.7 & 111 & 0.3 & -0.1 \\
\hline 10 & 44 & 46.2 & 44.7 & 82 & -0.9 & 1.8 \\
\hline 11 & 39 & 38.1 & 37.7 & 56 & 2.3 & 0.7 \\
\hline 12 & 8 & 8.2 & 7.4 & 49 & 1.2 & 1.6 \\
\hline 13 & 30 & 30.0 & 30.0 & 49 & 0.0 & 0.0 \\
\hline 14 & 49 & 48.9 & 50.0 & 51 & -2.0 & -2.2 \\
\hline 15 & 17 & 16.3 & 15.7 & 51 & 2.5 & 1.2 \\
\hline 16 & 29 & 29.6 & 29.6 & 45 & -1.3 & 0.0 \\
\hline 17 & 40 & 37.9 & 38.0 & 43 & 4.7 & -0.2 \\
\hline 18 & 20 & 17.1 & 17.1 & 52 & 5.6 & 0.0 \\
\hline 19 & 14 & 14.2 & 13.3 & 47 & 1.5 & 1.9 \\
\hline 20 & 37 & 36.0 & 36.0 & 65 & 1.5 & 0.0 \\
\hline 21 & 24 & 24.5 & 24.5 & 51 & -1.0 & 0.0 \\
\hline 22 & 7 & 8.3 & 8.1 & 49 & -2.2 & 0.4 \\
\hline 23 & 23 & 23.0 & 23.0 & 34 & 0.0 & 0.0 \\
\hline 24 & 12 & 12.2 & 10.8 & 37 & 3.2 & 3.8 \\
\hline 25 & 53 & 53.1 & 53.1 & 62 & -0.2 & 0.0 \\
\hline 26 & 13 & 12.6 & 12.1 & 41 & 2.2 & 1.2 \\
\hline 27 & 27 & 27.4 & 27.4 & 40 & -1.0 & 0.0 \\
\hline 28 & 32 & 32.5 & 32.5 & 36 & -1.4 & 0.0 \\
\hline 29 & 44 & 41.7 & 41.8 & 45 & 4.9 & -0.2 \\
\hline 30 & 22 & 22.5 & 22.6 & 35 & -1.7 & -0.3 \\
\hline 31 & 24 & 23.8 & 24.4 & 24 & -1.7 & -2.5 \\
\hline 32 & 32 & 32.2 & 33.3 & 38 & -3.4 & -2.9 \\
\hline 33 & 37 & 36.6 & 36.6 & 54 & 0.7 & 0.0 \\
\hline 34 & 31 & 31.2 & 31.5 & 32 & -1.6 & -0.9 \\
\hline 35 & 19 & 18.9 & 18.9 & 38 & 0.3 & 0.0 \\
\hline
\end{tabular}

\section{III.B.3. Comparison of absolute dose calculation results between the algorithm and the TPS}

The differences between the calculated results from the proposed algorithm and those from the KonRad IMRT system are presented in Fig. 3 for 541 calculation points. Here, doses at single points are compared, so no $\gamma$ index is used and the dose gradient at the point is not taken into account in the comparison. Note that the kernel was normalized to a relative output factor of 1.000 for a field size of 10 $\times 10 \mathrm{~cm}^{2}$, which corresponds to an absolute dose value $D_{\mathrm{MU}}^{\text {ref }}$ of $0.830 \mathrm{cGy} / \mathrm{MU}$ for the $15 \mathrm{MV}$ photon beams at $15 \mathrm{~cm}$ depth in the Siemens linear accelerators.

The differences found could include contributions from both calculation algorithms, so in some circumstances, the potential errors could be added or canceled. The differences

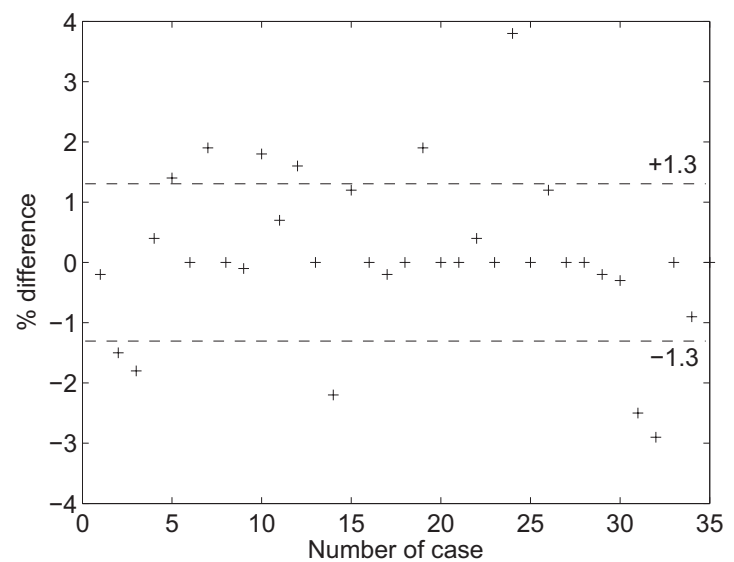

FIG. 2. Differences between the calculations of the presented algorithm and the measurements in 35 modulated fields. The dashed lines correspond to the averaged value \pm the standard deviation, so they encompass $66.7 \%$ of the data. 

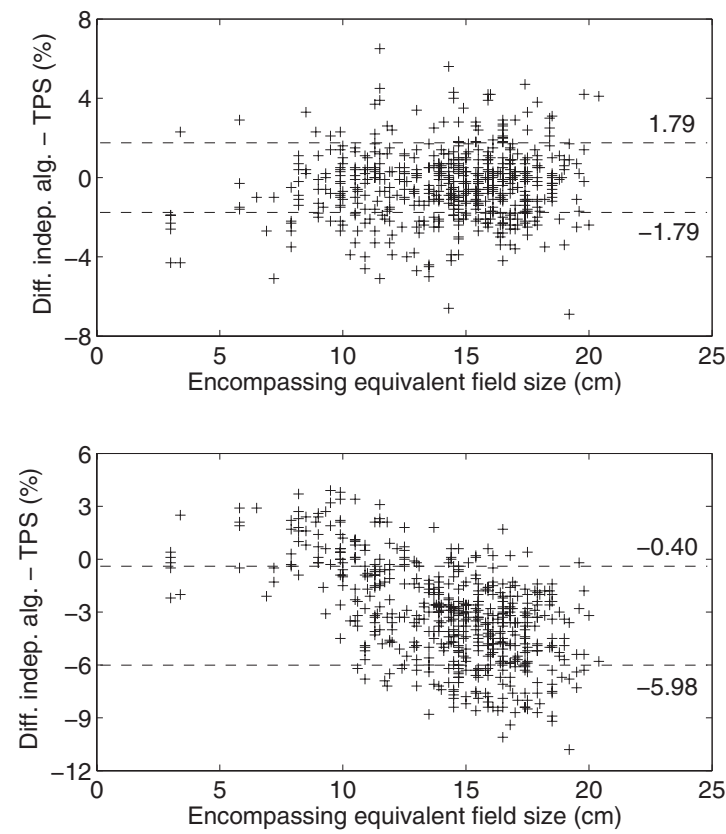

FIG. 3. Differences between the calculations of the presented algorithm and those from the KonRad TPS, as defined in Eq. (6), in 541 calculation points, with the corrected kernel (top) and the uncorrected (bottom). The differences are plotted versus the equivalent square field size side for the field that encompasses the complete set of segments for each IMRT field. With the uncorrected kernel, the mean of the differences is $-3.19 \%$. The dashed lines correspond to the averaged value \pm the standard deviation, so they encompass $66.7 \%$ of the data. The reduction in the standard deviation obtained with the correction is clearly shown (from 2.79 to 1.79 ).

between the calculations by the KonRad and the proposed algorithm have been calculated using the following expression:

$$
\% \operatorname{dif}=\frac{D_{\mathrm{alg}}-D_{\mathrm{KonRad}}}{D_{\text {Max Map }}},
$$

where $D_{\text {alg }}$ represents the dose calculated with the proposed algorithm and $D_{\text {KonRad }}$ is the dose calculated by the KonRad.
Obviously, the differences can increase at points where the dose is very low. Because small absolute differences can result in a large relative difference, the difference is related to the maximum value in the dose map ( $\left.D_{\text {Max Map }}\right)$.

The graph in Fig. 3 presents the differences between the results of the calculations using the proposed algorithm (corrected kernel at the top and uncorrected at the bottom) and using a commercial TPS. The mean value of all the differences with the corrected kernel is $-0.4 \%$. The negative sign means that the independent algorithm underestimates the absorbed dose with respect to the TPS. This mean difference is within the range of uncertainty in the determination of the absorbed dose under reference conditions. The differences range between $-6.9 \%$ and $6.5 \%$ and their standard deviation is $1.79 \%$. This result represents a clear improvement over the standard deviation of the differences obtained with the uncorrected kernel (Fig. 3, bottom) (2.79\%).

These fields can be composed of a number of segments that can be very different in size and shape. To represent the results, the differences for each IMRT field have been plotted relative to the side of the square field of equivalent size to the field that encompasses the complete set of segments for that modulated field. With the corrected kernel, the differences are not related to field size. Because most of the encompassing fields are rectangular instead of square, the classical formula has been used, which calculates the value of the equivalent field size side as $E_{Q}=4 A / P$, where $A$ is the area and $P$ the perimeter of the field.

It is interesting to analyze the differences between the calculated results of the proposed algorithm and those from the TPS by anatomical location. Table III displays the mean, standard deviation, and maximum and minimum differences for abdominal, pelvic, and head and neck treatment regions, and further subdivides these groups by the type of tumor (gastric, pancreatic, prostate, rectal, bladder, gynecologic, colon, brain, and head and neck tumors).

The table contains the modulated fields that have been

TABLE III. Differences and standard deviation between our calculations and those from the KonRad treatment planning system, grouped by anatomical localizations (bold). Below each localization, the different types of tumors are presented.

\begin{tabular}{cccccc}
\hline \hline & Nr. fields & $\begin{array}{c}\text { Mean diff. } \\
(\%)\end{array}$ & $\begin{array}{c}\text { STD } \\
(\%)\end{array}$ & $\begin{array}{c}\text { Max. } \\
(\%)\end{array}$ & $\begin{array}{c}\text { Min. } \\
(\%)\end{array}$ \\
\hline Abdominal & $\mathbf{4 2}$ & $\mathbf{0 . 4}$ & $\mathbf{1 . 7 9}$ & $\mathbf{4 . 5}$ & $-\mathbf{3 . 9}$ \\
Gastric & 21 & 0.4 & 1.50 & 4.2 & -2.6 \\
Pancreatic & 21 & 0.4 & 2.04 & 4.5 & -3.9 \\
Head and neck & $\mathbf{1 3 0}$ & $-\mathbf{0 . 6}$ & $\mathbf{1 . 6 9}$ & $\mathbf{3 . 9}$ & $-\mathbf{5 . 1}$ \\
Brain & 56 & -0.6 & 1.84 & 3.9 & -5.1 \\
Head and neck & 74 & -0.6 & 1.56 & 2.9 & -5.1 \\
& & & & & \\
Pelvis & $\mathbf{3 5 8}$ & $-\mathbf{0 . 4}$ & $\mathbf{1 . 8 0}$ & $\mathbf{6 . 5}$ & $-\mathbf{6 . 9}$ \\
Prostate & 119 & -0.8 & 2.21 & 6.5 & -6.9 \\
Rectum & 153 & -0.2 & 1.40 & 3.3 & -4.2 \\
Bladder & 42 & -0.4 & 1.89 & 4.2 & -6.6 \\
Gynecologic & 17 & -0.4 & 1.02 & 1.1 & -2.1 \\
Colon & 14 & -0.2 & 2.21 & 3.0 & -3.5 \\
\hline \hline
\end{tabular}




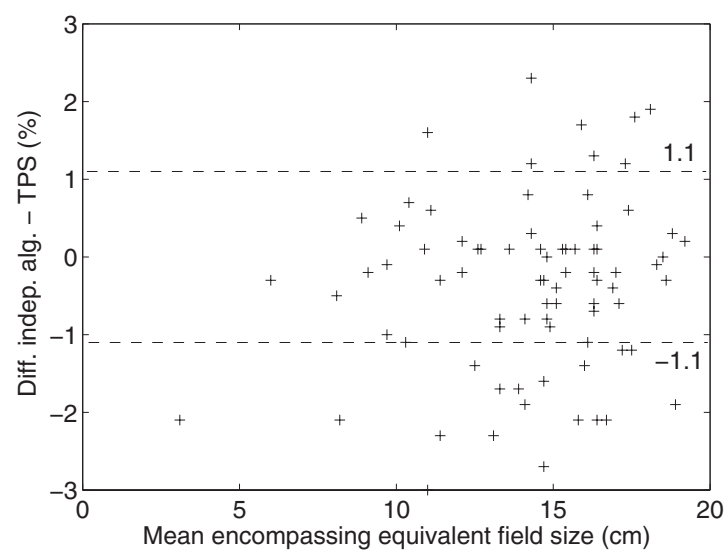

FIG. 4. Averaged differences per patient between the calculations of the presented algorithm and those from the KonRad TPS, from Siemens, in 79 patients. The dashed lines correspond to the averaged value \pm the standard deviation, so they encompass $66.7 \%$ of the data.

analyzed, with the exception of one plan for breast and one plan for mediastinum irradiation. Moreover, one plan for pelvic lymphoma and one plan for pelvic adenopathy are included in the pelvic statistics, but not in the subgroups. This is because subgroups containing only one case are not statistically significant.

It is apparent that the differences by type of tumor in a given anatomical location are minor, except for the prostate case, which differs from the remaining pelvic tumors on average $0.5 \%$ for the mean difference. Moreover, the mean differences between different locations vary within a $1 \%$ interval.

Finally, the mean differences by patient are presented as an absolute dose at single points as a function of the side of the equivalent-size square encompassing field (Fig. 4). All the 541 fields mentioned earlier correspond to 79 patients. The mean difference for each patient is shown, with a standard deviation of $1.1 \%$. The mean average difference is $-0.4 \%$. The averaged differences by patient lie in an interval between $-3.0 \%$ and $2.3 \%$.

\section{DISCUSSION}

The algorithm developed in Ref. 6 and improved in this study successfully uses an experimental kernel obtained by deconvolution. Another deconvolution procedure has also been investigated recently. Harder et al. ${ }^{11}$ have represented accurately, using a Lorentzian function, a pencil-beam kernel which is useful in small modulated fields. They have found good agreement between their results and the kernel proposed in Ref. 6. Both of these deconvolved kernels take into account the absence of electronic equilibrium as well as the partial obscuring of the primary extended source for small fields, which are two important effects that must be considered for accurate dose calculations. ${ }^{11,12}$

The kernel characterization procedure has been extended to correct the kernel tail that represents dose deposition by electrons far from the photon interaction point. With the previous formalism, accurate calculation of dose distributions was achieved. Now, with the new correction implemented for the experimental output-factor curve, the algorithm has been extended to predict properly the absolute dose calculations for all field sizes.

The measurements performed to test the accuracy of the algorithm provide confidence in the dose distributions as well as in the values of the absolute dose at single points. The $\gamma$ test showed that more than $99 \%$ of points passed the criteria for six of the seven cases studied. The calculation improves the results obtained with the uncorrected kernel, especially for modulated fields with large segments. The absolute dose calculation results also agree very well with the measured values. These data demonstrate the improvement of the method for IMRT dose calculations.

For testing the validity of this algorithm as a clinical tool for IMRT independent calculations, a set of absolute dose calculation results compared at single points is presented here. For the data presented, the differences are plotted versus the size of the field that encompasses the set of segments to demonstrate that the differences are independent of field size. However, calculations with the uncorrected kernel show differences related to field size. This is because of the "saturation" effect in the output-factor curve (Fig. 1). The previous algorithm was able to predict the absorbed dose for small field sizes, but the new improved kernel can perform these calculations for all field sizes because it takes proper account of the dose contribution from electrons coming from large distances. Furthermore, the differences are grouped by treatment site as well as by type of tumor. There are no relevant differences for any of the treatment sites except for the prostate cases, which deviate from the other pelvic tumors. However, the mean differences found among all types of tumors are within a $1 \%$ interval.

The agreement between the independent calculations and those from the TPS indicate that this method can be useful in clinical practice as a tool for individual patient IMRT QA. In the results presented here, the average differences with respect to measured values and the standard deviations of the differences are lower for the deconvolution algorithm, which uses a higher-resolution calculation grid. As for the comparison of the calculated results from the proposed algorithm with those from the TPS, the corrected kernel yields a smaller standard deviation. This is an important improvement with respect to the uncorrected kernel.

In addition to algorithm accuracy, calculation speed is also an important factor. For a seven-field IMRT dose plan, the time required to calculate the two-dimensional dose distributions is less than $1 \mathrm{~min}$ on a $3.06 \mathrm{GHz}$ Pentium 4 processor. This speed enables fast calculation of dose maps with $1 \mathrm{~mm}$ resolution that can be used for independent verification of TPS calculations. Moreover, the experimental kernel is independent of the Monte-Carlo-generated kernels commonly used by commercial TPS.

\section{CONCLUSIONS}

The capabilities of a pencil-beam calculation algorithm based on an experimental kernel obtained by deconvolution 
for dose calculation of IMRT plans have been improved to reproduce better the kernel tail and to predict the dose for all field sizes. Furthermore, this algorithm has been validated as an independent calculation procedure for everyday clinical practice.

The system presented here is completely independent of calculation algorithms based on Monte-Carlo-generated kernels and can calculate accurately in highly modulated fields. The experimental results demonstrate the capabilities of this algorithm for IMRT dose calculation as well as for IMRT dose plan verification.

The formalism developed in Ref. 6 and improved here is general. The kernel reconstructs the output-factor curve up to the largest fields available in the linear accelerator. However, in IMRT applications, the segment size used is often much smaller. The algorithm can be used as a double-check calculation formalism for IMRT, as well as for other applications. One remarkable property of the algorithm is its speed of calculation for multiple IMRT fields.

\section{ACKNOWLEDGMENTS}

This work was partially supported by the Spanish Ministerio de Educación y Ciencia, Project No. FIS 2008-01126. The authors also acknowledge an anonymous referee for the constructive criticism that motivated the improvement of the manuscript.

\footnotetext{
${ }^{a)}$ Electronic mail: jazcona@unav.es

${ }^{1}$ J. H. Kung, G. T. Y. Chen, and F. K. Kuchnir, "A monitor unit verification calculation in intensity modulated radiotherapy as a dosimetry quality assurance," Med. Phys. 27, 2226-2230 (2000).

${ }^{2}$ L. Xing, Y. Chen, G. Luxton, J. G. Li, and A. L. Boyer, "Monitor unit calculation for an intensity modulated photon field by a simple scattersummation algorithm," Phys. Med. Biol. 45, N1-N7 (2000).
}

${ }^{3}$ Y. Yang, L. Xing, J. G. Li, J. Palta, Y. Chen, G. Luxton, and A. Boyer, "Independent dosimetric calculation with inclusion of head scatter and MLC transmission for IMRT,” Med. Phys. 30, 2937-2947 (2003).

${ }^{4}$ Y. Watanabe, "Point dose calculations using an analytical pencil beam kernel for IMRT plan checking," Phys. Med. Biol. 46, 1031-1038 (2001).

${ }^{5}$ C. Chui, T. LoSasso, and A. Palm, A Practical Guide to Intensity Modulated Radiation Therapy (Medical Physics, Madison, 2003).

${ }^{6}$ J. D. Azcona and J. Burguete, "A system for intensity modulated dose plan verification based on an experimental pencil beam kernel obtained by deconvolution," Med. Phys. 35, 248-259 (2008).

${ }^{7}$ D. A. Low, W. B. Harms, S. Mutic, and J. A. Purdy, "A technique for the quantitative evaluation of dose distributions," Med. Phys. 25, 656-661 (1998).

${ }^{8} \mathrm{C}$. Chui and R. Mohan, "Extraction of pencil beam kernels by the deconvolution method," Med. Phys. 15, 138-144 (1988).

${ }^{9}$ T. C. Zhu, A. Ahnesjö, K. L. Lam, X. A. Li, C. M. Ma, J. R. Palta, M. B. Sharpe, B. Thomadsen, and R. C. Tailor, "Report of AAPM Therapy Physics Committee Task Group 74: In-air output ratio, $S_{c}$, for megavoltage photon beams," Med. Phys. 36, 5261-5300 (2009).

${ }^{10}$ I. J. Das, G. X. Ding, and A. Ahnesjö, "Small fields: Nonequilibrium radiation dosimetry," Med. Phys. 35, 206-215 (2008).

${ }^{11}$ A. Djouguela, D. Harder, R. Kollhoff, S. Foschepoth, W. Kunth, A. Rühmann, K. Willborn, and B. Poppe, "Fourier deconvolution reveals the role of the Lorentz function as the convolution kernel of narrow photon beams," Phys. Med. Biol. 54, 2807-2827 (2009).

${ }^{12}$ P. Caprile and G. H. Hartmann, "Development and validation of a beam model applicable to small fields," Phys. Med. Biol. 54, 3257-3268 (2009).

${ }^{13}$ U. Jeleń, M. Söhn, and M. Alber, "A finite size pencil beam for IMRT dose optimization," Phys. Med. Biol. 50, 1747-1766 (2005).

${ }^{14} \mathrm{U}$. Jeleń and M. Alber, "A finite size pencil beam algorithm for IMRT dose optimization: Density corrections," Phys. Med. Biol. 52, 617-633 (2007).

${ }^{15}$ IAEA, "Commissioning and quality assurance of computerized planning systems for radiation treatment of cancer," IAEA Technical Report Series No. 430 (International Atomic Energy Agency, Vienna, Austria, 2004).

${ }^{16}$ B. Fraass, K. Doppke, M. Hunt, G. Kutcher, G. Starkschall, R. Stern, and J. Van Dyke, "American Association of Physicists in Medicine Radiation Therapy Committee Task Group 53: Quality assurance for clinical radiotherapy treatment planning," Med. Phys. 25, 1773-1829 (1998). 